\title{
PRIMARY SJÖGREN SYNDROME AND NEUROLOGICAL AFFECTION: A CASE REPORT
}

Gabriele Souza Moulin Vago ${ }^{1, \star}$, Caroline Inez Fernandes Bulhão ${ }^{1}$, Juliana D’Agostino Gennari ${ }^{1}$, Dawton Yukito Torigoe ${ }^{1}$

1.Santa Casa de São Paulo, São Paulo (SP), Brazil.

*Corresponding author: gabrielemoulin.reumato@gmail.com

\section{BACKGROUND}

The primary Sjögren's syndrome is a chronic, immune-mediated disease. It evolves slowly and progressively and it results from the interaction between environmental and genetic factors. It is characterized morphologically by focal lymphoplasmacytic infiltration in the exocrine glands and in other epithelial tissues, causing dryness of the mucosa and large diversity of clinical and systemic manifestations. It is among the three most common autoimmune systemic diseases and it is the second most common rheumatic disease after rheumatoid arthritis. Its clinical picture is responsible for considerable impact on the quality of life of the patients. It is predominant in women, with a 9:1 ratio of women to men, having peak incidence in people aged between 50 and 60 years old; it is rare in children and young people. There are two forms of presentation: the primary form - when it occurs in isolation; and the secondary form - when it is associated with other autoimmune diseases, such as rheumatoid arthritis, systemic lupus erythematosus, polymyositis, autoimmune hepatitis, primary biliary cholangitis. This work aimed to present and discuss the clinical case of a young adult female with Sjögren's syndrome and neuromyelitis optica spectrum disorders.

\section{CASE REPORT}

The report of the case was collected in São Paulo on December 17th. The patient in question had been previously diagnosed with primary Sjögren's syndrome based on the 2016 ACR/EULAR classification criteria, with positive Anti-Ro antibody and positive Schirmer test ( $\geq 4$ points). Moreover, she presented negative AQP4-IgG (Aquaporin-4 antibody). The patient evolved with symptoms of neurological affection, such as hypoesthesia, hypersalivation and later dysphagia, nystagmus and ataxia. When being hospitalized, one year after the primary Sjögren's syndrome diagnosis, the patient presented as oligosymptomatic in relation to the sicca symptoms, with no significant complaints of xerophthalmia or xerostomia. The patient underwent therapy with methylprednisolone for five days and then maintenance therapy with prednisone and hydroxychloroquine, showing good responses until hospital discharge. She was followed up with infusions of rituximab

\section{CONCLUSION}

These procedures are according to other results described in the literature, which prove the clinical safety and efficacy of the use of rituximab in patients with primary Sjögren's syndrome and neurological affection. 